\title{
Clinical usefulness of fluorodeoxyglucose-positron emission tomography/computed tomography and carbohydrate antigen 19-9 in patients with periampullary tumors
}

Jeong Eon Kim, Min Ho Shin, Nam Kyu Choi

Division of Hepatobiliary-Pancreas and Liver Transplantation Surgery, Department of Surgery, Chosun University Hospital, Gwangju, Korea

Purpose: The prognosis of periampullary cancer varies with its origin and early diagnosis influences outcome. Endoscopic ultrasound, computed tomography, fine needle aspiration, and fluorodeoxyglucose-positron emission tomography/computed tomography $\left({ }^{18} \mathrm{FDG}-\mathrm{PET} / \mathrm{CT}\right)$ are helpful for diagnosis. This study evaluates the diagnostic efficacy of ${ }^{18} \mathrm{FDG}$-PET for preoperative periampullary tumors.

Methods: A series of 100 patients undergoing ${ }^{18} \mathrm{FDG}-\mathrm{PET} / \mathrm{CT}$ before surgical resection of periampullary tumors between March 2011 and February 2019 were enrolled. Maximum standardized uptake value (SUVmax) and carbohydrate antigen 19-9 (CA19-9) levels were compared with pathohistological confirmation of periampullary tumors.

Results: The SUVmax and uptake range varied with the origin of the periampullary tumors. The SUVmax was not available for 17 of the 42 pancreas tumors, three of 30 common bile duct tumors, and four of 18 ampulla of Vater tumors. The median SUVmax was 0.0 in benign tumors and 5.05 in malignant tumors. The mean SUVmax was $4.1 \pm 5.6$ in pancreatic tumors, $3.9 \pm 2.4$ in ampulla of Vater, and $6.0 \pm 3.7$ in common bile duct. The SUVmax was higher in common bile duct tumors than others. CA19-9 level was of diagnostic value in pancreatic tumor patients. The median CA19-9 levels were $7.64 \mathrm{U} / \mathrm{mL}$ (range, 2.71-45.05 U/mL) in benign tumors and 91.97 U/mL (range, 26.91-276.60 U/ $\mathrm{mL}$ ) in cancers patients.

Conclusion: Preoperative SUVmax and CA19-9 level were of diagnostic value for periampullary tumors originating in the pancreas.

Keywords: Pancreas neoplasm, Positron emission tomography computed tomography, Fluorodeoxyglucose F18, CA-19-9 antigen

\section{INTRODUCTION}

Periampullary tumors can arise from structures near the ampulla of Vater, pancreas, common bile duct, or duodenum, and the prog-

Received: Sep 24, 2019 Revised: Nov 19, 2019 Accepted: Dec 5, 2019

Correspondence to: Nam Kyu Choi

Division of Hepatobiliary-Pancreas and Liver Transplantation Surgery,

Department of Surgery, Chosun University Hospital, 365 Pilmun-daero,

Dong-gu, Gwangju 61453, Korea

Tel: +82-62-220-3965, Fax: +82-62-226-6515

E-mail: cnk@chosun.ack.kr

ORCID: Jeong Eon Kim (https://orcid.org/0000-0003-2678-8433), Min Ho Shin (https://orcid.org/0000-0001-9706-4366), Nam Kyu Choi (https://orcid.org/00000003-0726-1353)

Copyright @ 2019 Korean Society of Surgical Oncology

This is an Open Access article distributed under the terms of the Creative Commons Attribution Non-Commercial License (http://creativecommons.org/licenses/by-nc/4.0) which permits unrestricted non-commercial use, distribution, and reproduction in any medium, provided the original work is properly cited. nosis varies with the origin [1]. These neoplasms are usually treated by pylorus-preserving pancreatoduodenectomy or Whipple procedure. Pancreas cancer is one of most aggressive malignancies and cancers involving the pancreas are the fifth most common cause of cancer deaths in Korea [2]. Early diagnosis and differentiation of malignant from benign periampullary neoplasm is essential for decisions on performing surgical resection. The initial work up for a patient with periampullary masses includes computed tomography (CT), magnetic resonance imaging (MRI), endoscopic ultrasound (EUS), endoscopic ultrasound-guided fine needle aspiration (EUS-FNA), and magnetic resonance cholangiopancreatography (MRCP) [3]. FNA is important in the diagnosis of cystic lesions of the pancreas, but EUS-FNA of pancreatic tumors is difficult to perform because of the retroperitoneal location $[4,5]$. Invasive procedures like endoscopic retrograde cholangiopancreatography (ERCP) or EUS-FNA have diagnostic accuracy, but are associated with procedure-related complications [6]. ${ }^{18} \mathrm{FDG}$-PET/ 
CT a noninvasive imaging method that identifies areas of increased glucose metabolism. The maximum standardized uptake value (SUVmax) of ${ }^{18} \mathrm{FDG}$-PET/CT is useful for the diagnosis of malignant tumors and metastatic disease [7]. Carbohydrate antigen 19-9 (CA19-9), pathologic T stage, tumor size, lymphovascular invasion, lymph node metastasis and resection margin are prognostic values in periampullary cancer, but most are available only after surgical resection [4]. Preoperative CA19-9 is useful for the diagnosis, determining tumor stage, monitoring therapeutic efficacy and detecting tumor recurrence [8]. Previous studies support the clinical utility of CA19-9 and PET/CT for the diagnosis of periampullary tumors and found correlation with pathological characteristics between SUVmax and CA19-9 level in pancreatic tumors $[9,10]$. This study was a retrospective review and analysis of the association of ${ }^{18}$ FDG-PET SUVmax, CA19-9 level, and the characteristics of pathologically confirmed periampullary tumors.

\section{METHODS}

A total of 100 patients with ${ }^{18} \mathrm{FDG}$-PET/CT for diagnosis of a suspected periampullary cancer between March 2011 and February 2019 at the Chosun University Hospital were enrolled, and 90 with histologically confirmed periampullary tumor after respective surgery were included in the analysis.

Patients' work-up data from physical examinations, serologic studies, dynamic abdominopelvic CT and ${ }^{18}$ FDG-PET imaging were included on analysis. Eight patients with multiple metastases and two patients with carcinomatosis peritonei were exclude. Neuroendocrine tumor and intraductal papillary mucinous tumor neoplasm were included. Focal ${ }^{18} \mathrm{FDG}$ uptake was considered positive for malignancy and the SUVmax was calculated. Medical history, complete blood cell count, serum chemistry (aspartate aminotransferase, alanine aminotransferase, total bilirubin, lipase, amylase, $\gamma$-glutamyl transpeptidase), serum tumor markers such as carcinoembryonic antigen (CEA), CA19-9, and $\alpha$-fetoprotein (AFP) were available. The study was approved by the Institutional Review Board of Chosun University Hospital (IRB No. CHOSUN 2019-07-012) and performed in accordance with the principles of the Declaration of Helsinki. Written informed consent was waived.

\section{${ }^{18} \mathrm{FDG}-\mathrm{PET} / \mathrm{CT}$}

The patients fasted for at least 6 hours before PET/CT scan and serum glucose was tested to confirm a concentration below $180 \mathrm{mg} /$ day. Approximately $370 \mathrm{MBq}{ }^{18} \mathrm{FDG}$ was administered via the antecubital vein 60 minutes before starting the PET/CT scan. Wholebody coverage included the subcranial to the upper thigh regions. PET acquisition was preceded by low-dose contrast non-enhanced
CT followed by contrast-enhanced CT. A GE Healthcare Discovery ST (Chicago, IL, USA) was used for ${ }^{18}$ FDG-PET/CT imaging. The SUVmax indicates the maximum rate of metabolic glucose uptake. It was calculated in a circular region of interest in a transaxial image with the highest tumor uptake [10]. ${ }^{18} \mathrm{FDG}$ is a positron-emitting isotope and malignant cells, which are more metabolically active than benign cells, accumulate more glucose than other tissues and become FDG-avid [11].

\section{Statistical analysis}

Variables were expressed as means \pm standard deviation, medians, frequencies, and percentages.

The variables were analyzed by Kruskal-Wallis test. The diagnostic efficacy of ${ }^{18}$ FDG-PET/CT was indicated by the SUVmax observed by in periampullary cancers. The significance of differences in SUVmax and CA19-9 levels in benign and malignant tumor was determined with the Mann-Whitney nonparametric test when the values were not normally distributed. Statistical analysis was performed with SPSS version .25.0 (IBM Corp., Armonk, NY, USA).

\section{RESULTS}

Forty-seven of the 90 patients were included in the analysis; 43 were women and 47 were men with periampullary lesions. Forty-two of the tumors were in the pancreas, 30 were in the common bile duct, and 18 were in the ampulla of Vater (Table 1). The mean patient age was $67 \pm 10.2$ (range, 23-85 years). Resective surgery

Table 1. Tumor location

\begin{tabular}{ll}
\hline Location & No. (\%) \\
\hline Ampulla of Vater & $18(20.0)$ \\
Common bile duct & $30(33.3)$ \\
Pancreas & $42(46.7)$ \\
Total & $90(100)$ \\
\hline
\end{tabular}

Table 2. Surgical procedures

\begin{tabular}{lc}
\hline Procedure & No. (\%) \\
\hline PPPD & $59(65.6)$ \\
Distal pancreatectomy & $19(21.1)$ \\
Total pancreatectomy & $6(6.7)$ \\
Ampullectomy & $3(3.3)$ \\
Cholecystectomy \& CBD segmental resection, hepaticojejunostomy & $2(2.2)$ \\
Palliative Roux-en Y GJ & $1(1.1)$ \\
Total & $90(100)$ \\
\hline
\end{tabular}

PPPD, pylorus-preserving pancreatoduodenectomy; CBD, common bile duct; Roux-en Y GJ, Roux-en Y gastrojejunostomy. 
Table 3. Characteristic of patients

\begin{tabular}{lcccc}
\hline Variable & Pancreas & CBD & AoV & P-value \\
\hline Age (yr) & $65.6 \pm 12.0$ & $69.3 \pm 8.3$ & $70.6 \pm 7.8$ & 0.083 \\
Sex (male/female) & $22 / 20$ & $19 / 11$ & $6 / 12$ & 0.100 \\
Tumor size (cm) & & & & $2.1 \pm 1.2$ \\
$\quad$ Pathological & $3.1 \pm 1.6$ & $3.0 \pm 3.7$ & $0.6 \pm 0.7$ & 0.036 \\
CT & $2.9 \pm 0.4$ & $1.6 \pm 0.6$ & $3.9 \pm 2.4$ & 0.010 \\
SUVmax & $4.1 \pm 5.6$ & $6.0 \pm 3.7$ & $147.7 \pm 528.8$ & 0.035 \\
BMI (kg/m) & $22.7 \pm 3.3$ & $22.5 \pm 3.7$ & $138.9 \pm 136.8$ & 0.174 \\
CA19-9 (U/mL) & $149.4 \pm 237.4$ & $476.8 \pm 1.433 .0$ & $2.3 \pm 1.2$ & 0.039 \\
AFP (ng/mL) & $2.3 \pm 1.6$ & $1.9 \pm 0.9$ & $140.1 \pm 182.4$ & 0.803 \\
Amylase (U/L) & $83.4 \pm 104.9$ & $183.4 \pm 270.7$ & 0.143 \\
Total bilirubin (mg/dL) & $2.2 \pm 3.8$ & $5.9 \pm 6.6$ & $3.1 \pm 3.2$ & 0.136 \\
AST (U/L) & $58.1 \pm 96.2$ & $96.9 \pm 76.1$ & $138.9 \pm 258.5$ & 0.221 \\
ALT (U/L) & $92.3 \pm 281.4$ & $132.2 \pm 122.8$ & 0.069 \\
r-GTP (U/L) & $206.3 \pm 379.8$ & $553.5 \pm 505.6$ & $507.4 \pm 493.8$ & 0.294 \\
\hline
\end{tabular}

Values are presented as mean \pm standard deviation or number.

CBD, common bile duct; AoV, ampulla of Vater; CT, computed tomography; SUVmax, maximum standardized uptake value; BMI, body mass index; CA19-9, carbohydrate antigen 19-9; AFP, alpha fetoprotein; AST, aspartate aminotransferase; ALT, alanine aminotransferase; $\gamma$-GTP, $\gamma$-glutamyl transpeptidase.

Table 4. Pathological staging

\begin{tabular}{lc}
\hline Pathology & No. of patient (\%) \\
\hline Benign & $17(18.9)$ \\
T1 & $16(17.8)$ \\
T2 & $21(23.3)$ \\
T3 & $29(32.2)$ \\
T4 & $4(4.4)$ \\
X & $3(3.3)$ \\
Total & $90(100)$ \\
\hline
\end{tabular}

was performed in all patients under general anesthesia. Pylorus-preserving pancreatoduodenectomy was performed in 59 patients, 19 underwent distal pancreatectomy for pancreas tail cancers, and six underwent total pancreatectomy. Ampullectomy was performed for three ampulla of Vater tumors. Two patients with common bile duct tumors underwent bile duct resection and hepaticojejunostomy and one patient underwent Roux-en Y anastomosis and hepaticojejunostomy as palliative treatment (Table 2). The mean of CA19-9 levels were $149.4 \pm 237.4 \mathrm{U} / \mathrm{mL}$ in pancreas, $476.8 \pm 1,433.0 \mathrm{U} / \mathrm{mL}$ in common bile duct, and $138.9 \pm 136.8 \mathrm{U} /$ $\mathrm{mL}$ in ampulla of Vater tumors. AFP concentrations were $2.3 \pm 1.6$ $\mathrm{ng} / \mathrm{mL}$ in pancreas, $1.9 \pm 0.9 \mathrm{ng} / \mathrm{mL}$ in common bile duct, and 2.3 $\pm 1.2 \mathrm{ng} / \mathrm{mL}$ in ampulla of Vater tumors. CT estimated size was 2.9 $\pm 0.4 \mathrm{~cm}$ in pancreas, $1.6 \pm 0.6 \mathrm{~cm}$ in common bile duct, and $0.6 \pm$ 0.7 in ampulla of Vater tumors. The sizes determined by pathological evaluation were $3.1 \pm 1.6 \mathrm{~cm}$ in pancreas, $3.0 \pm 3.7 \mathrm{~cm}$ in common bile duct, $2.1 \pm 1.2 \mathrm{~cm}$ in ampulla of Vater tumors. The mean SUVmax was $4.1 \pm 5.6$ in pancreas, $6.0 \pm 3.7$ in common bile duct,
Table 5. Comparison of benign and malignancy tumors

\begin{tabular}{llcc}
\hline & \multicolumn{1}{c}{ Benign } & Malignancy & P-value \\
\hline SUVmax & $0.00(0.00-0.00)$ & $5.05(3.75-6.75)$ & 0.037 \\
CA19-9 $(\mathrm{U} / \mathrm{mL})$ & $7.64(2.71-45.05)$ & $91.97(26.91-276.60)$ & 0.038 \\
AFP $(\mathrm{ng} / \mathrm{mL})$ & $1.60(1.30-2.72)$ & $1.94(1.19-2.74)$ & 0.026 \\
CT-tumor size $(\mathrm{cm})$ & $2.80(2.00-3.00)$ & $12.00(1.20-3.00)$ & 0.037 \\
\hline
\end{tabular}

Values are presented as median (interquartile range).

SUVmax, maximum standardized uptake value; CA19-9, carbohydrate antigen 19-9; AFP, alpha fetoprotein; CT, computed tomography.

$3.9 \pm 2.4$ in ampulla of Vater tumors (Table 3).

\section{Pathological diagnosis}

This study used American Joint Committee on Cancer Staging Manual 8th edition. The pathology results are shown in Table 4. Pathological evaluation confirmed benign tumors in 17 patients and malignant tumors in 70 . Most benign lesions were in pancreas. Sixteen patients were TNM staging T1, 21 were T2, 29 were T3, and four were T4. Three patients who underwent palliative surgery had no pathological results (Table 4). The SUVmax (0.00 vs. 5.05) and CA19-9 levels (7.64 vs. 91.97) were higher in patients with malignant tumors than those with benign tumors. High CA19-9 levels and SUVmax were more common in patients with malignancies (Table 5).

\section{DISCUSSION}

Periampullary tumors arise from the ampulla of Vater, pancreas, 
common bile duct, or duodenum. Those that arise in the pancreas are lethal, mortality closely parallels incidence. As most patients with pancreatic cancer remain asymptomatic until the disease reaches an advanced stage, there is a need for screening patients at risk of periampullary cancers. Imaging is very important in the workup of periampullary cancers and is noninvasive. Mohammad Alizadeh et al. [12] and Kamisawa et al. [13] both found that EUS and EUS-guided FNA had good diagnostic efficacy, $85 \%$ accuracy and high sensitivity for pancreatic for cancer. Ibrahim et al. [14] reported that EUS-guided FNA was not sufficiently sensitive to identify low-grade intraductal papillary mucinous neoplasm, mucinous cystic neoplasm, or serous cystadenoma. However, its specificity was high enough to allow clinical identification of patients with high-grade dysplasia and/or coexistent carcinoma; EUS-guided FNA of pancreatic cysts does have real clinical value. The diagnostic accuracy of EUS-guided FNA makes useful as an adjunct to CT/MRI in determining the resectability of pancreatic cancer. However, EUS-guided FNA and ERCP are invasive and are associated with complications such as bleeding, tissue damage, and pancreatitis [15]. The use of PET and PET/CT for the initial staging and treatment planning has been valid [16]. The ${ }^{18}$ FDG uptake pattern can differentiate malignant from benign pancreatic masses, though a recent study failed to determine a sufficiently specific and sensitive SUVmax cutoff value because the pancreatic tuberculosis lesions had very high ${ }^{18} \mathrm{FDG}$ uptake [17]. Nasr Shaban [1] reported that ${ }^{18} \mathrm{FDG}$-PET/CT significantly increased the ability $(\mathrm{P}=0.025)$ to detect of pancreatic cancer and extra abdominal recurrences. Sun et al. [10] found that SUVmax and CA19-9 significantly improved the sensitivity and specificity of the diagnosis of pancreatic cancer and evaluation of prognosis. Burge et al. [18] reported that inflammation increased FDG uptake in nonmalignant tissue, but was useful for surgical planning because it was able to detect metastasis. Jha and Bijan [19] reviewed the use of PET/CT in the evaluation of pancreatic cancer. The value of PET/CT for the of periampullary tumors has not been established. Akcam et al. [20] reported that PET/CT was not useful for the diagnosis of tumors originating in the ampullary region, and that low or normal SUVmax may indicate the presence of a periampullary mass. Verma et al. [15] reported that PET scans provide information on occult metastasis, but its clinical benefit in diagnosis is not yet established. In this study, the SUVmax was elevated in pathologically confirmed periampullary lesions but the mean SUVmax and range varied in different periampullary tumors depending on the origin. The SUVmax ranged from 0 to 25.6 for pancreatic tumors, and 0 to 7.1 in both ampulla of Vater and distal common bile duct. The SUVmax was higher in pancreatic tumors than other malignant periampullary tumors. However, 17 of the 42 pancreatic tumors, three of 30 common bile duct tumors, four of 18 ampulla of Vater tumors did not have SUVmax. CA19-9 is a diagnostic serum marker of pancreatic cancer in symptomatic patients and preoperative serum CA19-9 levels have prognostic value in pancreatic cancer patients with nonspecific symptoms or signs. However, malignancies have false-negative results because of genotype and increased false positive results in the obstructive jaundice [21]. In this study, CA19-9 levels were higher in patients with pancreatic lesions than in those with ampulla of Vater or common bile duct lesions.

The study limitations include limited data on the association of SUVmax and tumor differentiation, and not including perineural invasion and differentiation, but on pathological characteristics. The retrospective design was subject to patient selection bias. Finally, presurgical CA19-9 data was lacking.

In conclusion, preoperative PET/CT and SUVmax were useful for the evaluation of patients with periampullary tumors. Elevated SUVmax is associated with malignancy in common bile duct. CA19-9 was of diagnostic value in patients with pancreatic tumors. The SUVmax and CA19-9 level would be useful in planning surgical treatment of patients with periampullary tumors.

\section{CONFLICT OF INTEREST}

No potential conflict of interest relevant to this article was reported.

\section{ACKNOWLEDGMENTS}

This study was supported by research funds from Chosun University Hospital.

\section{REFERENCES}

1. Nasr Shaban EAI. Added value of 18 -F-FDG-PET/CT in patients with pancreatic cancer: initial observation. Egypt J Radiol Nucl Med 2016;47:1275-82.

2. Jung KW, Won YJ, Kong HJ, Lee ES. Prediction of cancer incidence and mortality in Korea, 2018. Cancer Res Treat 2018;50:317-23.

3. Pimiento JM, Davis-Yadley AH, Kim RD, Chen DT, Eikman EA, Berman CG, et al. Metabolic activity by 18F-FDG-PET/CT Is prognostic for stage I and II pancreatic cancer. Clin Nucl Med 2016;41:177-81.

4. Im HJ, Oo S, Jung W, Jang JY, Kim SW, Cheon GJ, et al. Prognostic value of metabolic and volumetric parameters of preoperative FDG-PET/CT in patients with resectable pancreatic cancer. Medicine (Baltimore) 2016;95:e3686.

5. Chirindel A, Alluri KC, Chaudhry MA, Wahl RL, Pawlik TM, Her- 
man JM, et al. Prognostic value of FDG PET/CT-Derived parameters in pancreatic adenocarcinoma at initial PET/CT staging. AJR Am J Roentgenol 2015;204:1093-9.

6. Cheng MF, Wang HP, Tien YW, Liu KL, Yen RF, Tzen KY, et al. Usefulness of PET/CT for the differentiation and characterization of periampullary lesions. Clin Nucl Med 2013;38:703-8.

7. Koyama K, Okamura T, Kawabe J, Nakata B, Chung KH, Ochi H, et al. Diagnostic usefulness of FDG PET for pancreatic mass lesions. Ann Nucl Med 2001;15:217-24.

8. Kau SY, Shyr YM, Su CH, Wu CW, Lui WY. Diagnostic and prognostic values of CA 19-9 and CEA in periampullary cancers. J Am Coll Surg 1999;188:415-20.

9. Ballehaninna UK, Chamberlain RS. The clinical utility of serum CA 19-9 in the diagnosis, prognosis and management of pancreatic adenocarcinoma: an evidence based appraisal. J Gastrointest Oncol 2012;3:105-19.

10. Sun Y, Duan Q, Wang S, Zeng Y, Wu R. Diagnosis of pancreatic cancer using ${ }^{18}$ F-FDG PET/CT and CA19-9 with SUVmax association to clinical characteristics. J BUON 2015;20:452-9.

11. Kawada N, Uehara H, Hosoki T, Takami M, Shiroeda H, Arisawa T, et al. Usefulness of dual-phase 18F-FDG PET/CT for diagnosing small pancreatic tumors. Pancreas 2015;44:655-9.

12. Mohammad Alizadeh AH, Shahrokh S, Hadizadeh M, Padashi M, Zali MR. Diagnostic potency of EUS-guided FNA for the evaluation of pancreatic mass lesions. Endosc Ultrasound 2016;5:30-4.

13. Kamisawa T, Wood LD, Itoi T, Takaori K. Pancreatic cancer. Lancet 2016;388:73-85.

14. Ibrahim AA, Cramer HM, Wu HH. Endoscopic ultrasound-guid- ed fine-needle aspiration of the pancreas: a retrospective study of 1000 cases. J Am Soc Cytopathol 2014;3:227-35.

15. Verma A, Shukla S, Verma N. Diagnosis, preoperative evaluation, and assessment of resectability of pancreatic and periampullary cancer. Indian J Surg 2015;77:362-70.

16. Dibble EH, Karantanis D, Mercier G, Peller PJ, Kachnic LA, Subramaniam RM. PET/CT of cancer patients: part 1, pancreatic neoplasms. AJR Am J Roentgenol 2012;199:952-67.

17. Santhosh S, Mittal BR, Bhasin D, Srinivasan R, Rana S, Das A, et al. Role of (18)F-fluorodeoxyglucose positron emission tomography/ computed tomography in the characterization of pancreatic masses: experience from tropics. J Gastroenterol Hepatol 2013;28:25561.

18. Burge ME, O’Rourke N, Cavallucci D, Bryant R, Francesconi A, Houston K, et al. A prospective study of the impact of fluorodeoxyglucose positron emission tomography with concurrent non-contrast CT scanning on the management of operable pancreatic and peri-ampullary cancers. HPB 2015;17:624-31.

19. Jha P, Bijan B. PET/CT for pancreatic malignancy: potential and pitfalls. J Nucl Med Technol 2015;43:92-7.

20. Akcam AT, Ulku A, Rencuzogulları A, Parsak CK, Yapar Z, Doran F, et al. 18F-FDG activitiy PET/CT and CA-19.9 levels for the prediction of histopathological features and localization of peri- ampullary tumors. Turk J Gastroenterol 2015;26:170-5.

21. Bottger T, Hassdenteufel A, Boddin J, Kuchle R, Junginger T, Prellwitz W. Value of the CA 19-9 tumor marker in differential diagnosis of space-occupying lesions in the head of the pancreas. Chirurg 1996;67:1007-11. 